Deutsche Kartographie Kongress, den wir im Jahr 2019 wiederum gemeinsam mit der Kongressmesse Intergeo in Stuttgart durchführen werden, wird sich thematisch an diesen Herausforderungen orientieren.

Über die Entwicklungen in Kartographie und Geomatik, über aktuelle Forschungsergebnisse, praktische Anwendungen, neue Produkte und neue Verwaltungsdienstleistungen berichtet die allem im Flaggschiff der deutschsprachigen Kartographie, der KN. Die DGfK hat nach langer interner Diskussion für die KN einen ternational Publishing AG beschlossen, insbesondere um die Weiterentwicklung und das Fortbestehen des Wissenschaftsteils der KN zu sichern. Damit einher geht nun der Abschied vom Kirschbaum Verlag nach 45-jähriger vertrauensvoller Zusammenarbeit, der vor allem die in der langjährigen Zusammenarbeit engagierten Mitglieder der DGfK nicht unberührt lässt. Die beiden Editoren Mark Vetter und Rolf Harbeck haben den Dank der Schriftleitung zum Ausdruck gebracht und die gemeinsame $\mathrm{Ar}$ beit im Rückblick gewürdigt.

Mir bleibt an dieser Stelle, dem Kirschbaum Verlag und Herrn Bernhard Kirschbaum mit seinem Team auch im Namen aller Vereinsmitglieder und der Leserinnen und Leser der $\mathrm{KN}$ zu danken. Wir wünschen dem Verlag und seinem Leiter und Lenker für die der Kirschbaum Verlag wird durch den digitalen Wandel in eine neue Epoche geführt werden müssen. vents- und Weihnachtszeit wünsche ich Ihnen, liebe Leserinnen und Leser, nun Ruhe und Muße und einen guten Start ins neue Jahr 2019. Ich hoffe, viele von Ihnen zum Neujahrsempfang der DGfK und der Sektion BerlinBrandenburg am 17. Januar 2019 in der Staatsbibliothek zu Berlin DGfK in ihren Publikationen, vor Verlagswechsel zur Springer InZukunft alles Gute, denn auch

Für die vor uns liegende Ad- wiederzutreffen, zu dem wir mit Gerd Buziek den Leiter der Arbeitsgruppe Geoinformation des Branchenverbandes Bitkom gewinnen konnten. Zudem werden wir die Veranstaltung in bewährter Weise gemeinsam mit der Society for News Design wieder mit einem Workshop zu den Themen News-Infographics-AnalyticsMaps verbinden.

Manfred Weisensee, Hude

Präsident der DGfK -

Gesellschaft für Kartographie und Geomatik

\section{DGfK-Sektion Dresden}

\section{Aus der Arbeit der} Sektion 2017/2018

\section{Vortragsveranstaltungen und Exkursionen}

Das Veranstaltungsprogramm 2017/18 begann im Oktober 2017 mit dem Vortrag von Ulrich Schumacher (Leibniz-Institut für ökologische Raumentwicklung Dresden) über „Eine Kartenserie zur Physiognomie des Urbanen“, welche von ihm als ein Beitrag zu dem Buch „Stadt im Spannungsfeld von Kompaktheit, Effizienz und Umweltqualität“ (erschienen 2017 bei Springer Spektrum) vorgestellt wurde. Im November rückte die Geodateninfrastruktur der Stadt Leipzig in den Fokus. Zu Gast war Jana Dietrich als Leiterin des Amtes für Geoinformation und Bodenordnung, die über aktuelle Problemstellungen und Lösungen auf diesem Fachgebiet unterrichtete.

Die alljährliche Mitgliederversammlung fand traditionell im Rahmen der Weihnachtsfeier im Dezember statt. Der Vortrag unseres neuen Ehrenmitglieds Dr. Werner Stams, langjähriger Inhaber der Dozentur für Kartengestaltung am Institut für Kartographie der TU Dresden und Autor zahlreicher kartographiehistorischer Werke, rundete den Abend mit dem Vortrag „Meine Lehre zum Kartolithographen 19421946 in Leipzig“" ab.
Roland Mau vom Bundesamt für Kartographie und Geodäsie stellte uns zu Beginn des Jahres 2018 die digitalen Landschaftsmodelle DLM250 und DLM1000 vor. Im Februar referierte der hiesige Sektionsleiter, Mathias Gröbe von der TU Dresden, über die Fusion von Daten von OpenStreetMap und Wikidata zur Herstellung von Karten.

Ebenfalls im Februar fand unsere Exkursion nach Berlin ins Naturkundemuseum sowie die Kartensammlung der Staatsbibliothek zu Berlin statt. In der frisch renovierten Bibliothek führte uns Steffi Mittenzwei u. a. ins Tresormagazin und zeigte uns einige Schätze der Sammlung.

Den März nutzte die Sektion für einen Besuch der IABG Geodatenfactory Dresden. Hier wurden wir über die Arbeitsfelder der IABG informiert und ausführlich in die angewandten Methoden der Luftbildauswertung an verschiedenen Beispielen eingeführt.

Im April fand ein viertägiger Workshop an der TU Dresden statt, zu dem sich 20 Teilnehmer unterschiedlichster Altersstufen aus ganz Deutschland angemeldet hatten. Themen waren $u$. a. die kartographische Geländedarstellung, OpenStreetMap und die Auswertung von Daten aus sozialen Medien. Inhaltlich abgerundet wurde die Veranstaltung durch Wolfgang Puschs Vortrag „Reliefs - Anachronismus oder Geovisualisierung mit Zukunft?"

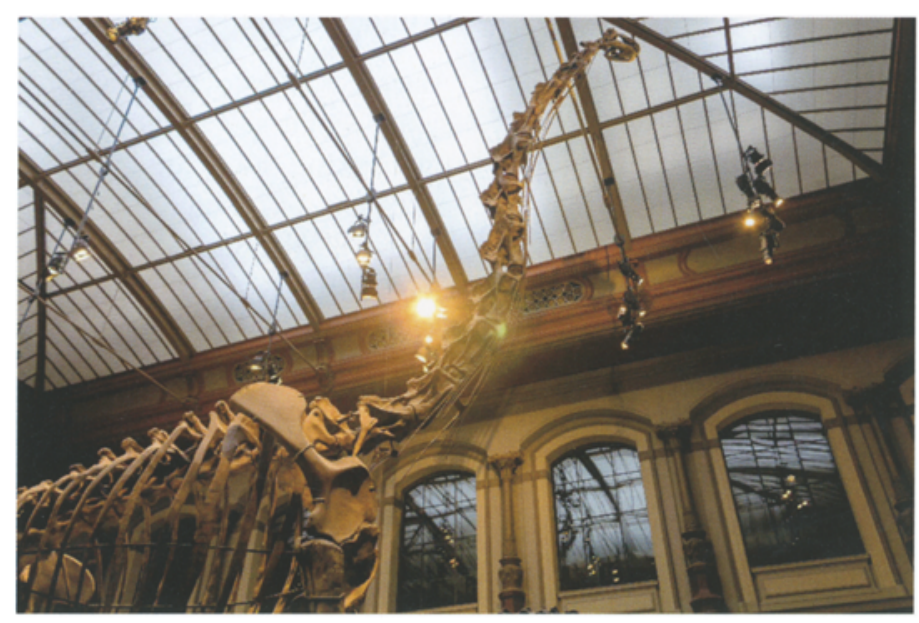

Impression aus dem Naturkundemuseum in Berlin (Foto: Mathias Gröbe)

über die Herstellung von Bergmodellen.

Über die Erstellung und Laufendhaltung der Namibia-Karte informierte uns Prof. Uwe Ulrich Jäschke von der HTW Dresden im Mai. Im Juni folgte dann der letzte Vortrag zum Thema „Das Individuum im Raum“ von Sebastian Meier aus Berlin.

Anfang September ließen wir das Veranstaltungsjahr bei einem geselligen Grillabend am Institut für Kartographie an der TU Dresden ausklingen, da wir leider unser über die Jahre liebgewonnenes Domizil im Bootshaus an der Elbe nicht mehr anmieten konnten.

Neue Webseite und neuer Workshop

Passend zum Start ins neue Veranstaltungsprogram hat unser Schriftführer Steffen Hild unsere Webseite neu gestaltet. Von der alten Adresse werden Sie automatisch auf die neue Seite unter https://dresden.dgfk.net/ weitergeleitet. Hier finden Sie aktuelle Informationen zu allen Veranstaltungen und Hintergrundinformationen zur Sektion.

Vom 11. bis 13. Februar 2019 wird erneut ein kartographischer Workshop an der TU Dresden stattfinden. Weitere Informationen finden Sie auf der Webseite http://workshop.dgfk-dresden.de/.

Mathias Gröbe, Leiter der DGfKSektion Dresden 